\title{
An immersed boundary/level-set method for incompressible viscous flows in complex geometries with good conservation properties
}

\author{
Yoann Cheny — Olivier Botella \\ LEMTA, Nancy-Université, CNRS \\ 2, avenue de la Forêt de Haye, BP 160 \\ F-54504 Vandouvre-lès-Nancy \\ \{Yoann.Cheny, Olivier.Botella\}@ensem.inpl-nancy.fr
}

ABSTRACT. This paper concerns the development of a new Cartesian grid / immersed boundary (IB) method for the computation of incompressible viscous flows in irregular geometries. In IB methods, the computational grid is not aligned with the irregular boundary, and of upmost importance for accuracy and stability is the discretization in cells which are "cut" by the boundary. In this paper, we present an IB method (the LS-STAG method) based on the Cartesian MAC method where the irregular boundary is represented by its level-set function. This implicit representation of the immersed boundary enables us to discretize efficiently the fluxes in the cut-cells by imposing the strict conservation of total kinetic energy at the discrete level. The accuracy and robustness of our method are assessed on benchmark flows.

RÉSUMÉ. Nous présentons une nouvelle méthode cartésienne de type "frontière immergée 》 (immersed boundary, ou méthode IB) pour les écoulements visqueux incompressibles en géométries irrégulières, qui est telle que la grille de calcul n'est pas alignée avec la frontière de la géométrie. Notre méthode IB, appellée LS-STAG, utilise une fonction level-set pour représenter les frontières irrégulières. Cette représentation implicite de la frontière nous permet de discrétiser efficacement les flux dans les cellules cartésiennes coupées en imposant que le schéma numérique conserve l'énergie cinétique totale au sens discret. La précision et la robustesse de la méthode sont évaluées pour des écoulements canoniques.

KEYWORDS: incompressible viscous flows, immersed boundary methods, level-set methods.

MOTS-CLÉS : fluides visqueux incompressibles, frontières immergées, méthodes «level-set».

DOI:10.3166/EJCM.18.561-587 @ 2009 Lavoisier, Paris

EJCM - 18/2009. High-performance computing, pages 561 to 587 


\section{Introduction}

Much attention has recently been devoted to the extension of Cartesian grid flow solvers to complex geometries by immersed boundary (IB) methods (see Mittal et al. (2005) for a recent review). In these methods, the irregular boundary is not aligned with the computational grid, and the treatment of the cut-cells, cells of irregular shape which are formed by the intersection of the Cartesian cells by the immersed boundary, remains an important issue. Indeed, the discretization in these cut-cells should be designed such that : (a) the global stability and accuracy of the original Cartesian method are not severely diminished and $(b)$ the high computational efficiency of the structured solver is preserved.

Two major classes of IB methods can be distinguished on the basis of their treatment of cut-cells. Classical IB methods such as the momentum forcing method introduced by Fadlun et al. (2000), use a finite volume/difference structured solver in Cartesian cells away from the irregular boundary, and discard the discretization of flow equations in the cut-cells. Instead, special interpolations are used for setting the value of the dependent variables in the latter cells. Thus, strict conservation of quantities such as mass, momentum or kinetic energy is not observed near the irregular boundary. The most severe manifestations of these shortcomings is the occurrence of non-divergence free velocities or unphysical oscillations of the pressure in the vicinity of the immersed boundary. Numerous revisions of these interpolations are still proposed for improving the accuracy and consistency of this class of IB methods (Kang et al., 2004; Muldoon et al., 2008).

A second class of IB methods (also called cut-cell methods or simply Cartesian grid methods (Ye et al., 1999; Dröge et al., 2005; Chung, 2006; Mittal et al., 2008) aims for actually discretizing the flow equations in cut-cells. The discretization in the cut-cells is usually performed by ad hoc treatments which have more in common with the techniques used on curvilinear or unstructured body-conformal grids than Cartesian techniques. Most notable is the cell merging technique used by Ye et al. (1999) and Chung (2006) that merges a cut-cell with a neighboring Cartesian cell to form a new polygonal cell with more than four neighbors. The discretization stencil in this newly formed cell loses thus the 5-point structure (in 2D) of Cartesian methods. Such treatments of the cut-cells generate a non negligible bookkeeping to discretize the flow equations and actually solve them, and it is difficult to evaluate the impact of these treatments on the computational cost of the flow simulations.

The purpose of this article is to present a new IB method for incompressible viscous flows which takes the best aspects of both classes of IB methods. This method, called the LS-STAG method, is based on the symmetry preserving finite-volume method by Verstappen et al. (2003), which has the ability to preserve on non-uniform staggered Cartesian grids the conservation properties (for total mass, momentum and kinetic energy) of the original MAC method (Harlow et al., 1965). The LS-STAG method has the following distinctive features : 
- The immersed boundary is implicitly represented by its signed distance function, also known as the level-set function (Osher et al., 2003). Level-set methods were devised by Osher et al. (1988) for the solution of computational physics problems involving dynamic interfaces. So far for incompressible flows, the main application areas of level set methods have been the computation of two-phase flows (Sussman et al., 1994). In the present paper, the level-set function enables us to easily compute all relevant geometry parameters of the computational cells, reducing thus the bookkeeping associated to the handling of complex geometries.

- In contrast to classical IB methods, flow variables are actually computed in the cut-cells, and not interpolated. Furthermore, the LS-STAG method has the ability to discretize the fluxes in Cartesian and cut-cells in a consistent and unified fashion : there is no need for deriving an ad hoc treatment for the cut-cells, which would be totally disconnected from the basic MAC discretization used in the Cartesian cells.

- For building our discretization, we have required the strict conservation of total kinetic energy in the whole fluid domain, which is a crucial property for obtaining physically realistic numerical solutions (Morinishi et al., 1998; Verstappen et al., 2003). To achieve this property up to the cut-cells, we had to precisely take into account the terms acting on the immersed boundary in the global conservation equations, at both continuous and discrete levels. As a result, the convective and pressure fluxes have been unambiguously determined by these requirements, and the boundary conditions at the immersed boundary have been incorporated into these fluxes with a consistent manner.

- From the algorithmic point of view, one of the main consequences is that the LSSTAG discretization preserves the 5-point structure of the original Cartesian method. This property allowed the use of an efficient black box multigrid solver for structured grids (van Kan et al., 2000), where no ad hoc modifications had to be undertaken for taking account of the immersed boundary.

The paper is organized as follows. In Section 2, we first recall the notations and salient properties of the staggered Cartesian mesh and then we present the LS-STAG mesh, its extension for the handling of immersed boundaries. Next, we will present discretization of the continuity equation, whose consistency in both cut- and Cartesian cells is a crucial point for building an energy preserving numerical method for incompressible flows. In the next subsections, we will impose kinetic energy conservation upon our numerical scheme for completely characterizing the discrete pressure and convective fluxes in the cut-cells. We will complete the spatial discretization with a discretization of the viscous fluxes in the cut-cells that will preserve the simplicity of the 5-point stencil of Cartesian methods. Finally, Section 3 is devoted to numerical tests on canonical flows at low to moderate Reynolds number for assessing the accuracy and robustness of the LS-STAG method: the Couette Taylor flow and the flow past a circular cylinder. 


\section{Preliminaries and description of the LS-STAG method in complex geometries}

In the following, we consider discretizing the Navier-Stokes equations with the finite-volume method in a rectangular domain $\Omega$, where $\Gamma$ is its surface. After integration by parts of these equations in $\Omega$ (or in any of its closed subdomains), the integral form of the continuity equation reads :

$$
\int_{\Gamma} \boldsymbol{v} \cdot \boldsymbol{n} \mathrm{d} S=0
$$

where $\boldsymbol{v}=(u, v)$ is the velocity, and the integral form of the momentum equations in the $x$ and $y$ directions is respectively :

$$
\begin{aligned}
& \frac{\mathrm{d}}{\mathrm{d} t} \int_{\Omega} u \mathrm{~d} V+\int_{\Gamma}(\boldsymbol{v} \cdot \boldsymbol{n}) u \mathrm{~d} S+\int_{\Gamma} p \boldsymbol{e}_{x} \cdot \boldsymbol{n} \mathrm{d} S-\frac{1}{\operatorname{Re}} \int_{\Gamma} \nabla u \cdot \boldsymbol{n} \mathrm{d} S=0, \\
& \frac{\mathrm{d}}{\mathrm{d} t} \int_{\Omega} v \mathrm{~d} V+\int_{\Gamma}(\boldsymbol{v} \cdot \boldsymbol{n}) v \mathrm{~d} S+\int_{\Gamma} p \boldsymbol{e}_{y} \cdot \boldsymbol{n} \mathrm{d} S-\frac{1}{\operatorname{Re}} \int_{\Gamma} \boldsymbol{\nabla} v \cdot \boldsymbol{n} \mathrm{d} S=0,
\end{aligned}
$$

where $p$ is the pressure and Re is the Reynolds number.

\subsection{The staggered MAC mesh for Cartesian geometries}

The Cartesian method on which our IB method is based is the second-order finite volume discretization of Verstappen et al. (2003), which has the ability to preserve on non-uniform Cartesian cells the conservation properties (for total mass and kinetic energy) of the original MAC method on a uniform staggered grid (Harlow et $a l ., 1965)$. The staggered arrangement of the unknowns in a Cartesian cell is represented in Figure 1. The rectangular computational $\Omega$ is partitioned into Cartesian cells $\left.\Omega_{i, j}=\right] x_{i-1}, x_{i}[\times] y_{j-1}, y_{j}\left[\right.$, whose volume is $V_{i, j}=\Delta x_{i} \Delta y_{j}$ and center is $\boldsymbol{x}_{i j}^{\mathrm{c}}=\left(x_{i}^{\mathrm{c}}, y_{j}^{\mathrm{c}}\right)$. The surface $\Gamma_{i, j}$ of cell $\Omega_{i, j}$ is subdivided into four elementary plane faces as :

$$
\Gamma_{i, j}=\Gamma_{i, j}^{\mathrm{e}} \cup \Gamma_{i, j}^{\mathrm{w}} \cup \Gamma_{i, j}^{\mathrm{n}} \cup \Gamma_{i, j}^{\mathrm{s}},
$$

by using the usual compass notations (Patankar, 1980). Cell $\Omega_{i, j}$ is used as a control volume for discretizing the continuity Equation [1], whereas the staggered cell $\Omega_{i, j}^{u}=$ ]$x_{i}^{\mathrm{c}}, x_{i+1}^{\mathrm{c}}[\times] y_{j-1}, y_{j}[$ is the control volume for the $x$-momentum Equation [2a]. For subdividing the surface $\Gamma_{i, j}^{u}$ of this control volume, we first decompose the north and south faces of $\Omega_{i, j}$ as $\Gamma_{i, j}^{\mathrm{n}}=\Gamma_{i, j}^{\mathrm{n}, \mathrm{w}} \cup \Gamma_{i, j}^{\mathrm{n}, \mathrm{e}}$ and $\Gamma_{i, j}^{\mathrm{s}}=\Gamma_{i, j}^{\mathrm{s}, \mathrm{w}} \cup \Gamma_{i, j}^{\mathrm{s}, \mathrm{e}}$ respectively, and then write :

$$
\Gamma_{i, j}^{u}=\Gamma_{i, j}^{u, \mathrm{w}} \cup \Gamma_{i, j}^{u, \mathrm{e}} \cup\left(\Gamma_{i, j}^{\mathrm{s}, \mathrm{e}} \cup \Gamma_{i+1, j}^{\mathrm{s}, \mathrm{w}}\right) \cup\left(\Gamma_{i, j}^{\mathrm{n}, \mathrm{e}} \cup \Gamma_{i+1, j}^{\mathrm{n}, \mathrm{w}}\right) .
$$

An analogous decomposition holds for the faces of the control volume $\Omega_{i, j}^{v}$, which is used for discretizing the $y$-momentum Equation [2b]. These notations will be useful for describing the LS-STAG mesh. 


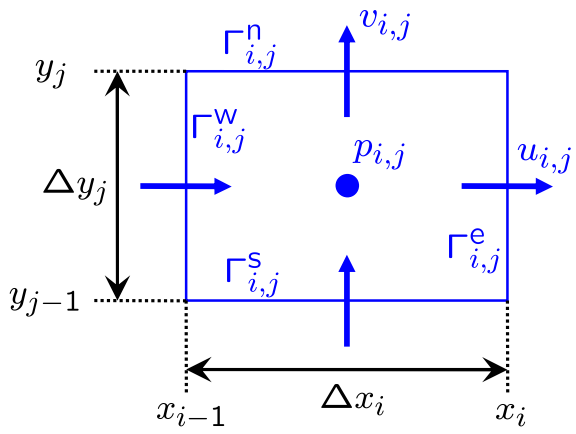

(a)

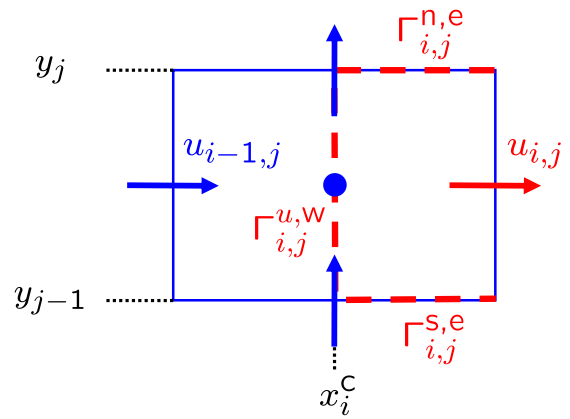

(b)

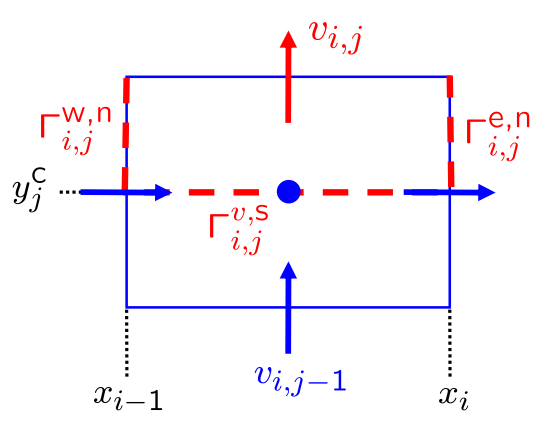

(c)

Figure 1. Staggered arrangement of the variables for (a) : a Cartesian cell $\Omega_{i, j}$, and representation of control volumes for $(b): u_{i, j}$ and $(c): v_{i, j}$. The control volumes $\Omega_{i, j}^{u} \in \Omega_{i, j} \cup \Omega_{i+1, j}$ and $\Omega_{i, j}^{v} \in \Omega_{i, j} \cup \Omega_{i, j+1}$ are to be completed with their complementary part in $\Omega_{i+1, j}$ and $\Omega_{i, j+1}$ respectively

\subsection{The LS-STAG mesh for irregular geometries}

We consider now an irregular solid domain $\Omega^{\text {ib }}$ which is embedded in the computational domain $\Omega$, such that $\Omega^{\mathrm{f}}=\Omega \backslash \Omega^{\mathrm{ib}}$ represents the fluid domain where the Navier-Stokes equations are to be discretized. To keep track of the irregular boundary $\Gamma^{\mathrm{ib}}$, we employ a signed distance function $\phi(\boldsymbol{x})$ (i.e. , the level set function (Osher et al., 2003)) such that $\phi(\boldsymbol{x})$ is negative in the fluid region $\Omega^{\mathrm{f}}, \phi(\boldsymbol{x})$ is positive in the solid region $\Omega^{\mathrm{ib}}$, and such that the boundary $\Gamma^{\mathrm{ib}}$ corresponds to the zero level-set of this function, i.e. :

$$
\phi(x) \equiv\left\{\begin{aligned}
-\Delta, & x \in \Omega^{\mathrm{f}}, \\
0, & \boldsymbol{x} \in \Gamma^{\mathrm{i}}, \\
+\Delta, & \boldsymbol{x} \in \Omega^{\mathrm{ib}},
\end{aligned}\right.
$$

where $\Delta$ represents the distance between $x$ and the nearest point on the immersed boundary. 


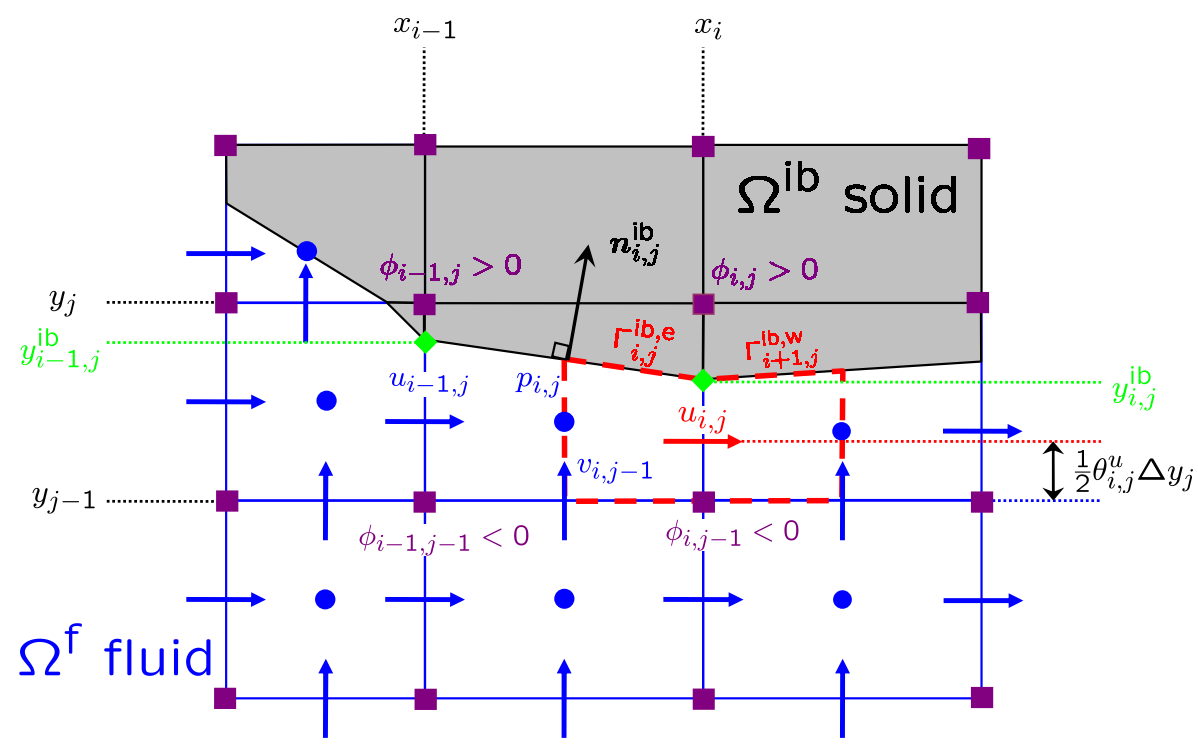

Figure 2. Staggered arrangement of the variables near the trapezoidal cut-cell $\Omega_{i, j}$ on the LS-STAG mesh

This leads to the modification of the MAC mesh that is described in Figure 2, and that will be subsequently referred to as the $L S$-STAG mesh. In each cut-cell $\Omega_{i, j}$, the immersed boundary is represented by a line segment whose extremities are defined by linear interpolation of the variable $\phi_{i, j}$, which takes the value of the level-set function $\phi\left(x_{i}, y_{j}\right)$ at the upper right corner of the cell. We use notations similar to the Cartesian method for the faces of the cut-cells. For example in Figure 2, the faces of the trapezoidal cut-cell $\Omega_{i, j}$ are denoted :

$$
\Gamma_{i, j}=\Gamma_{i, j}^{\mathrm{w}} \cup \Gamma_{i, j}^{\mathrm{e}} \cup \Gamma_{i, j}^{\mathrm{s}} \cup \Gamma_{i, j}^{\mathrm{ib}},
$$

where $\Gamma_{i, j}^{\mathrm{ib}}$ represents the solid north face of the cut-cell. As it will be justified later, the velocity unknowns are exactly located in the middle of the fluid part of the faces. In Figure 2, the discrete pressure $p_{i, j}$ is located at the intersection of the velocity locations. This location is used for visualization purpose only, and will never be used in the discretization. In effect, we will find out in Section 2.4 that the discrete pressure is piecewise constant in each cut-cell, as in some mixed finite element methods (e.g. Pironneau (1989)), and thus does not need to be precisely located in the cut-cells.

As observed in Figure 2, there are three basic types of cut-cells : trapezoidal cells such as $\Omega_{i, j}$ or $\Omega_{i+1, j}$, triangular cells (i.e., $\Omega_{i-1, j+1}$ ) and pentagonal cells (i.e.,$\Omega_{i-1, j}$ ). The discretization of the momentum equations will be performed in 


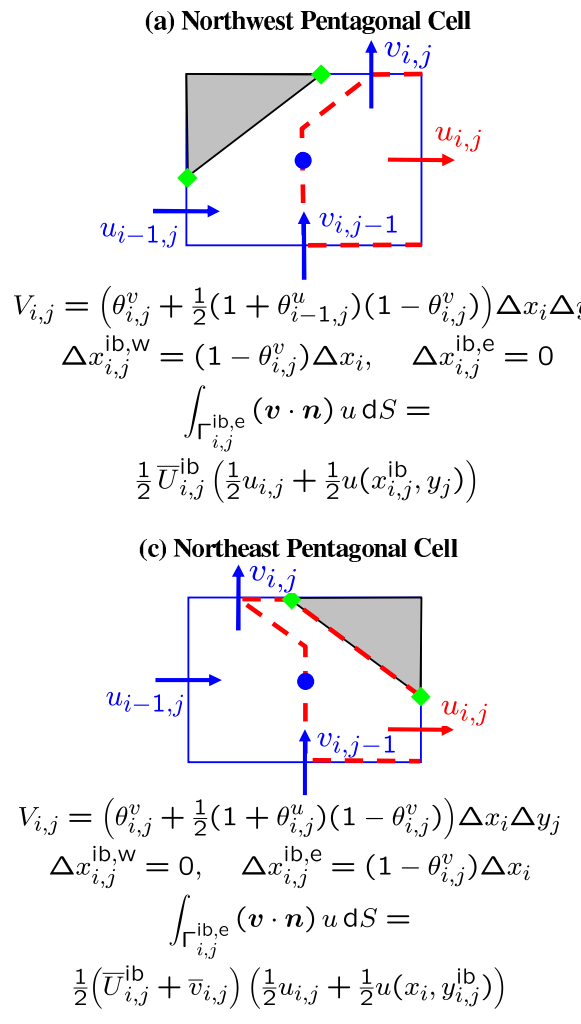

(e) Northwest Triangular Cell

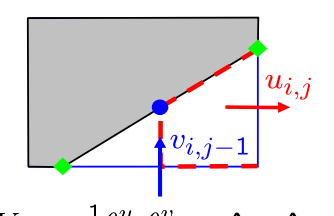

(b) West Trapezoidal Cell

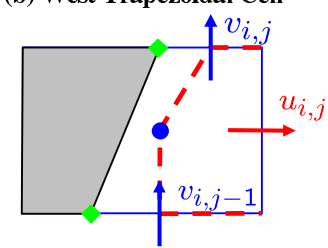

$$
\begin{gathered}
V_{i, j}=\frac{1}{2}\left(\theta_{i, j-1}^{v}+\theta_{i, j}^{v}\right) \Delta x_{i} \Delta y_{j} \\
\Delta x_{i, j}^{\mathrm{ib}, \mathrm{e}}=0 \\
\int_{\Gamma_{i, j}^{\mathrm{ib}, \mathrm{e}} \cup \Gamma_{i, j}^{u, \mathrm{w}}}(\boldsymbol{v} \cdot \boldsymbol{n}) u \mathrm{~d} S= \\
\frac{1}{2}\left(\bar{U}_{i, j}^{\mathrm{ib}}-\bar{u}_{i, j}\right)\left(\frac{1}{2} u_{i, j}+\frac{1}{2} u_{i, j}^{\mathrm{ib}}\right)
\end{gathered}
$$

\section{(d) North Trapezoidal Cell}

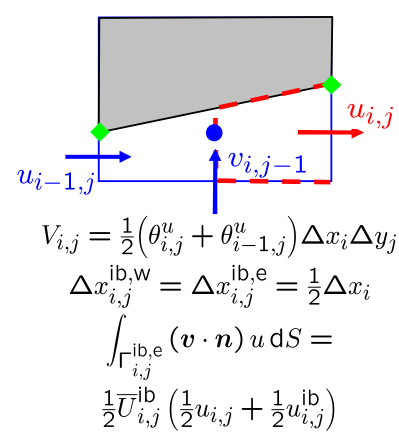

(f) Northwest Salient Corner

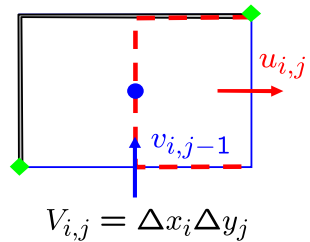

$$
\begin{gathered}
\int_{\Gamma_{i, j}^{\mathrm{ib}, \mathrm{e}} \cup \Gamma_{i, j}^{u, \mathrm{w}}}(\boldsymbol{v} \cdot \boldsymbol{n}) u \mathrm{~d} S= \\
\frac{1}{2}\left(u_{i, j}^{\mathrm{ib}}\left[n_{x} \Delta S\right]_{i, j}^{\mathrm{ib}}-\bar{u}_{i, j}\right)\left(\frac{1}{2} u_{i, j}+\frac{1}{2} u_{i, j}^{\mathrm{ib}}\right)+\frac{1}{2} v_{i, j}^{\mathrm{ib}}\left[n_{y} \Delta S\right]_{i, j}^{\mathrm{ib}}\left(\frac{1}{2} u_{i, j}+\frac{1}{2} u\left(x_{i}, y_{i, j}^{\mathrm{ib}}\right)\right)
\end{gathered}
$$

Figure 3. Basic types of half control volume for the velocity unknown $u_{i, j}$ inside the cut-cell $\Omega_{i, j}$, and relevant quantities used in the LS-STAG discretization. The diamonds $(\downarrow)$ denote the locations of the discretization of the velocity boundary conditions 
the staggered control volumes $\Omega_{i, j}^{u}$ and $\Omega_{i, j}^{v}$, whose shape has to be adapted to each type of cut-cells. For example in Figure 2, the faces of the control volume $\Omega_{i, j}^{u}$ read :

$$
\Gamma_{i, j}^{u}=\Gamma_{i, j}^{u, \mathrm{w}} \cup \Gamma_{i, j}^{u, \mathrm{e}} \cup\left(\Gamma_{i, j}^{\mathrm{s}, \mathrm{e}} \cup \Gamma_{i+1, j}^{\mathrm{s}, \mathrm{w}}\right) \cup\left(\Gamma_{i, j}^{\mathrm{ib}, \mathrm{e}} \cup \Gamma_{i+1, j}^{\mathrm{ib}, \mathrm{w}}\right),
$$

where the solid faces $\Gamma_{i, j}^{\mathrm{ib}, \mathrm{e}} \cup \Gamma_{i+1, j}^{\mathrm{ib}, \mathrm{w}}$ are formed with two halves of the solid face of the neighboring trapezoidal cut-cells $\Gamma_{i, j}^{\mathrm{ib}, \mathrm{e}} \subset \Gamma_{i, j}^{\mathrm{ib}}$ and $\Gamma_{i+1, j}^{\mathrm{ib}, \mathrm{w}} \subset \Gamma_{i+1, j}^{\mathrm{ib}}$. For the other type of cut-cells, these control volumes will be constructed from the six halves of generic control volumes that we represent in Figure $3{ }^{1}$. In this figure, the irregular shape of the staggered control volumes is given for representation purpose only, and their geometric parameters, such as their actual volume or shape of the vertical faces $\Gamma_{i, j}^{u, \mathrm{w}}$ and $\Gamma_{i, j}^{u, \mathrm{e}}$ are never used by the LS-STAG discretization : instead, we will employ arguments based on the strict conservation of global quantities of the flow, such as total mass and kinetic energy, for discretizing the Navier-Stokes equations in the cutcells. More precisely, we will built in the next section a discretization for each of the half control volumes of Figure 3, such that any combination of these halves that forms a control volume $\Omega_{i, j}^{u}$ yields a consistent discretization of the momentum equations, with the aforementioned global conservation properties.

However, the LS-STAG method relies on a sharp representation of the geometry of the cut-cells $\Omega_{i, j}$. In this respect, the level-set function will prove to be a very efficient tool for calculating the geometric parameters of a cut-cell, such as its volume or the projected areas of its faces. A quantity that will be extensively used for calculating these parameters is the fluid portion of the faces of cell $\Omega_{i, j}$. For example in Figure 2, by using one-dimensional linear interpolation of $\phi\left(x_{i}, y\right)$ for $y \in\left[y_{j-1}, y_{j}\right]$, we calculate the length $y_{i, j}^{\mathrm{ib}}-y_{j-1}$ of the portion of face $\Gamma_{i, j}^{\mathrm{e}}$ that belongs to the fluid domain as :

$$
y_{i, j}^{\mathrm{ib}}-y_{j-1}=\theta_{i, j}^{u} \Delta y_{j}, \quad \text { with } \theta_{i, j}^{u}=\frac{\phi_{i, j-1}}{\phi_{i, j-1}-\phi_{i, j}} \text { since } \phi\left(x_{i}, y_{i, j}^{\mathrm{ib}}\right)=0 .
$$

The scalar quantities $\theta_{i, j}^{u}$ and $\theta_{i, j}^{v}$, which take values in $[0,1]$, will subsequently be called the cell-face fraction ratios. They represent the fluid portion of the east and north faces $\Gamma_{i, j}^{\mathrm{e}}$ and $\Gamma_{i, j}^{\mathrm{n}}$ respectively. They will be extensively used for detecting if the discrete velocities $u_{i, j}$ and $v_{i, j}$ belong to the fluid domain, and for discretizing the surface and volume integrals in the Navier-Stokes Equations [1]-[2]. The cell-face fraction ratios also appear in the analytic expression of the volume $V_{i, j}$ of the cut-cells given in Figure 3.

1. Note that the re-entrant corner of a Cartesian mesh is a particular case of pentagonal cell (c) when $\phi_{i, j}=0$, and that Case (f) (which corresponds to $\phi_{i-1, j-1}=\phi_{i-1, j}=\phi_{i, j}=0$ and $\phi_{i, j-1}<0$ ) corresponds to the particular case of a salient corner. Only the definition of the cell volume distinguishes the latter cell from the limiting case of triangle (e) defined by $\phi_{i-1, j-1}=$ $\phi_{i, j}=0, \phi_{i-1, j}>0$ and $\phi_{i, j-1}<0$. Only the definition of the cell volume distinguishes the latter cell from the limiting case of triangle (e) defined by $\phi_{i-1, j-1}=\phi_{i, j}=0, \phi_{i-1, j}>0$ and $\phi_{i, j-1}<0$. 


\subsection{Discretization of the continuity equation}

As in the Cartesian method of Verstappen et al. (2003), the starting point of the LS-STAG discretization concerns the mass conservation law [1] in cell $\Omega_{i, j}$, which is represented by Equation [1]. For any fluid cell (cut-cell or Cartesian), we denote its faces as $\Gamma_{i, j}=\Gamma_{i, j}^{\mathrm{w}} \cup \Gamma_{i, j}^{\mathrm{e}} \cup \Gamma_{i, j}^{\mathrm{s}} \cup \Gamma_{i, j}^{\mathrm{n}} \cup \Gamma_{i, j}^{\mathrm{ib}}$, and decompose the continuity equation as the net mass flux through each of these faces :

$$
\dot{m}^{i, j} \equiv-\bar{u}_{i-1, j}+\bar{u}_{i, j}-\bar{v}_{i, j-1}+\bar{v}_{i, j}+\bar{U}_{i, j}^{\mathrm{ib}}=0 .
$$

In this equation, $\bar{U}_{i, j}^{\mathrm{ib}} \equiv \int_{\Gamma_{i, j}^{\mathrm{b} b}} \boldsymbol{v}^{\mathrm{ib}} \cdot \boldsymbol{n}_{i, j}^{\mathrm{ib}} \mathrm{d} S$ denotes the mass flow through the solid part of the cell boundary. This quantity may be non zero for non homogeneous boundary conditions only. The mass flow through the fluid part of the faces is denoted with a bar : for example, the flow through face $\Gamma_{i, j}^{e}$ of Figure 2 is :

$$
\bar{u}_{i, j} \equiv \int_{\Gamma_{i, j}^{\mathrm{e}}} \boldsymbol{v} \cdot \boldsymbol{e}_{x} \mathrm{~d} S=\int_{y_{j-1}}^{y_{i, j}^{\mathrm{ib}}} u\left(x_{i}, y\right) \mathrm{d} y .
$$

In order to easily discretize this integral, we first locate the discrete unknown $u_{i, j}$ in the middle of the fluid part of the face as :

$$
u_{i, j} \equiv u\left(x_{i}, y_{j-1}+\frac{1}{2} \theta_{i, j}^{u} \Delta y_{j}\right)
$$

Then, by using midpoint quadrature, we obtain :

$$
\bar{u}_{i, j} \cong \theta_{i, j}^{u} \Delta y_{j} u_{i, j}
$$

and following analogous discretizations for the other faces, the discrete continuity equation reads :

$$
\begin{array}{r}
\dot{m}^{i, j} \equiv \Delta y_{j}\left(\theta_{i, j}^{u} u_{i, j}-\theta_{i-1, j}^{u} u_{i-1, j}\right)+\Delta x_{i}\left(\theta_{i, j}^{v} v_{i, j}-\theta_{i, j-1}^{v} v_{i, j-1}\right) \\
+\bar{U}_{i, j}^{\mathrm{ib}}=0
\end{array}
$$

We now turn to the discretization of the boundary term as :

$$
\bar{U}_{i, j}^{\mathrm{ib}} \cong u_{i, j}^{\mathrm{ib}}\left[n_{x} \Delta S\right]_{i, j}^{\mathrm{ib}}+v_{i, j}^{\mathrm{ib}}\left[n_{y} \Delta S\right]_{i, j}^{\mathrm{ib}},
$$

where $\left[n_{x} \Delta S\right]_{i, j}^{\mathrm{ib}}$ and $\left[n_{y} \Delta S\right]_{i, j}^{\mathrm{ib}}$ are the projected areas of the solid face of the cut-cell in the horizontal and vertical directions respectively, and velocity $\boldsymbol{v}_{i, j}^{\mathrm{ib}}=\left(u_{i, j}^{\mathrm{ib}}, v_{i, j}^{\mathrm{ib}}\right)$ represents an approximation of the velocity on the solid boundary $\Gamma_{i, j}^{\mathrm{ib}}$ of the cut-cell. This last term is calculated with the trapezoidal rule, for example in Figure 2 :

$$
\boldsymbol{v}_{i, j}^{\mathrm{ib}}=\frac{1}{2} \boldsymbol{v}\left(x_{i}, y_{i, j}^{\mathrm{ib}}\right)+\frac{1}{2} \boldsymbol{v}\left(x_{i-1}, y_{i-1, j}^{\mathrm{ib}}\right),
$$


where the velocity datum at the intersection points between the solid boundary and the cut-cells is given by the boundary conditions of the continuous problem. The projected areas are readily calculated from the cell-face fraction ratios as :

$$
\left[n_{x} \Delta S\right]_{i, j}^{\mathrm{ib}}=\left(\theta_{i-1, j}^{u}-\theta_{i, j}^{u}\right) \Delta y_{j}, \quad\left[n_{y} \Delta S\right]_{i, j}^{\mathrm{ib}}=\left(\theta_{i, j-1}^{v}-\theta_{i, j}^{v}\right) \Delta x_{i}
$$

We mention that this discretization of the continuity equation is valid for any type of cut-cells, and in the particular case of a Cartesian fluid cell ( such as the cell-face fraction ratios are equal to 0 or 1 only), Equation [12] reduces to the discrete continuity equation of the original $M A C$ method.

In the following, it will be useful to write the discrete continuity equation in its matrix form :

$$
\mathcal{D} U+\bar{U}^{\mathrm{ib}}=0
$$

where each line $(i, j)$ of this system corresponds to Equation [12] written in cell $\Omega_{i, j}$, and the vectors $U$ and $\bar{U}^{\text {ib }}$ contains the velocity unknowns $\left(u_{i, j}, v_{i, j}\right)$ and the discretization of the boundary terms respectively.

\subsection{Energy preserving discretization of the momentum equations}

Now, we turn to the discretization of the momentum equations [2], whose semidiscrete matrix representation reads :

$$
\mathcal{M} \frac{\mathrm{d} U}{\mathrm{~d} t}+\mathcal{C}[\bar{U}] U+\mathcal{G} P-\frac{1}{\operatorname{Re}} \mathcal{K} U+S^{\mathrm{ib}, \mathrm{c}}-\frac{1}{\mathrm{Re}} S^{\mathrm{ib}, \mathrm{v}}=0
$$

where the diagonal mass matrix $\mathcal{M}$ is build from the volume of the fluid cells, matrix $\mathcal{C}[\bar{U}]$ represents the discretization of the convective fluxes, $\mathcal{G}$ is the discrete pressure gradient, $\mathcal{K}$ represents the viscous term, $S^{\mathrm{ib}, \mathrm{c}}$ and $S^{\mathrm{ib}, \mathrm{v}}$ are source terms arising from the boundary conditions of the convective and viscous terms respectively.

The discretization of these terms should preserve the discrete counterpart of the conservation of the kinetic energy $\mathrm{E}_{\mathrm{c}}(t)=\frac{1}{2} \int_{\Omega^{\mathrm{f}}} \boldsymbol{v}^{2} \mathrm{~d} V$ when the viscosity vanishes. The conservation equation for $\mathrm{E}_{\mathrm{c}}(t)$ is obtained from the Navier-Stokes equation by multiplying the momentum Equation [2] with $\boldsymbol{v}$ and integrating in the whole fluid domain. After integration by parts of the volumic integrals, this conservation equation reads

$$
\frac{\mathrm{dE}_{\mathrm{c}}}{\mathrm{d} t}=\int_{\Omega^{\mathrm{f}}}\left[\left(\frac{|\boldsymbol{v}|^{2}}{2}+p\right) \nabla \cdot \boldsymbol{v}-\frac{|\boldsymbol{v}|^{2}}{\operatorname{Re}}\right] \mathrm{d} V-\int_{\Gamma^{\mathrm{ib}}}\left(\frac{|\boldsymbol{v}|^{2}}{2}+p-\frac{\boldsymbol{\nabla} \boldsymbol{v}}{\operatorname{Re}}\right) \boldsymbol{v} \cdot \boldsymbol{n} \mathrm{d} S .
$$

Thanks to the continuity equation, the only term remaining in the volume integral involves the viscous stresses, and expresses the loss of energy by viscous dissipation. 
The non-vanishing terms in the surface integrals show that the pressure and convective terms only influence the kinetic energy budget by their action at the immersed boundary.

In order to build the discrete counterpart of [18], we first discretize the kinetic energy $E_{\mathrm{c}}(t)$ with the trapezoidal rule in each fluid cell $\Omega_{i, j}$ :

$$
\mathrm{E}_{\mathrm{c}}(t) \cong \mathrm{E}_{\mathrm{c}}^{h}(t)=\frac{1}{2} U^{\mathrm{T}} \mathcal{M} U+\frac{1}{2} U^{\mathrm{ib}, \mathrm{T}} \mathcal{M}^{\mathrm{ib}} U^{\mathrm{ib}},
$$

where $\mathcal{M}$ is the diagonal mass matrix that appears in the discrete momentum Equation [17], and $U^{\mathrm{ib}, \mathrm{T}} \mathcal{M}^{\mathrm{ib}} U^{\mathrm{ib}}$ is the contribution of the boundary conditions, which are assumed to be steady. For each line $(i, j)$ of the discrete system [17], the trapezoidal rule gives the value of the diagonal coefficient of the mass matrix in the horizontal and vertical directions :

$$
\left[\mathcal{M}^{x}\right]_{\mathrm{P}}(i, j)=\frac{1}{2} V_{i, j}+\frac{1}{2} V_{i+1, j}, \quad\left[\mathcal{M}^{y}\right]_{\mathrm{P}}(i, j)=\frac{1}{2} V_{i, j}+\frac{1}{2} V_{i, j+1} .
$$

These expressions show that, in the cut-cells at least, the mass matrix for $u_{i, j}$ and $v_{i, j}$ is not constructed from the actual areas of $\Omega_{i, j}^{u}$ et $\Omega_{i, j}^{v}$.

The conservation equation for $\mathrm{E}_{\mathrm{c}}^{h}(t)$ is obtained after time-differentiation of [19], then by using the discrete momentum Equation [17] we finally obtain :

$$
\frac{\mathrm{d} E_{\mathrm{c}}^{h}}{\mathrm{~d} t}=-U^{\mathrm{T}} \frac{\mathcal{C}[\bar{U}]^{\mathrm{T}}+\mathcal{C}[\bar{U}]}{2} U-P^{\mathrm{T}} \mathcal{G}^{\mathrm{T}} U-U^{\mathrm{T}} \frac{\mathcal{K}^{\mathrm{T}}+\mathcal{K}}{2 \mathrm{Re}} U-U^{\mathrm{T}}\left(S^{\mathrm{ib}, \mathrm{c}}-\frac{1}{\operatorname{Re}} S^{\mathrm{ib}, \mathrm{v}}\right) .
$$

In order to obtain an expression similar to [18], the viscous term $-U^{\mathrm{T}}\left(\mathcal{K}^{\mathrm{T}}+\mathcal{K}\right) U$ should mimic the viscous dissipation of the kinetic energy budget, and thus should always be strictly negative. This feature is obtained as soon as the matrix $\mathcal{K}^{\mathrm{T}}+\mathcal{K}$ is positive definite. For a finite-volume method, this is obtained as soon as the discrete viscous flux is stable and consistent (Eymard et al., 2000). Note that the symmetry of $\mathcal{K}$ is not required. If, in addition, we impose that the discretization of the convective terms leads to a skew-symmetric matrix :

$$
\mathcal{C}[\bar{U}]=-\mathcal{C}[\bar{U}]^{\mathrm{T}}
$$

and that, as in the finite element method, the pressure gradient is dual to the divergence operator (see Equation [16]) :

$$
\mathcal{G}=-\mathcal{D}^{\mathrm{T}}
$$

we finally observe that the boundary terms only affects the kinetic energy budget when the viscosity vanishes $(\operatorname{Re} \rightarrow+\infty)$ :

$$
\frac{\mathrm{dE}_{\mathrm{c}}^{h}}{\mathrm{~d} t}=-P^{\mathrm{T}} \bar{U}^{\mathrm{ib}}-U^{\mathrm{T}} S^{\mathrm{ib}, \mathrm{c}} .
$$




\section{Discretization of the pressure gradient}

In the above equation, the term $P^{\mathrm{T}} \bar{U}^{\mathrm{ib}}$ represents the discretization of the pressure term $\int_{\Gamma \text { ib }} p \boldsymbol{v} \cdot \boldsymbol{n} \mathrm{d} S$ in Equation [18], and condition [23] allows us to completely determine the discret pressure gradient in control volumes $\Omega_{i, j}^{u}$ and $\Omega_{i, j}^{v}$ from the discrete divergence operator [12] :

$$
\begin{gathered}
\int_{\Gamma_{i, j}^{u}} p \boldsymbol{e}_{x} \cdot \boldsymbol{n} \mathrm{d} S \cong\left[\mathcal{G}^{x} P\right]_{i, j}=\theta_{i, j}^{u} \Delta y_{j}\left(p_{i+1, j}-p_{i, j}\right), \\
\int_{\Gamma_{i, j}^{v}} p \boldsymbol{e}_{y} \cdot \boldsymbol{n} \mathrm{d} S \cong\left[\mathcal{G}^{y} P\right]_{i, j}=\theta_{i, j}^{v} \Delta x_{i}\left(p_{i, j+1}-p_{i, j}\right) .
\end{gathered}
$$

These formulae are valid for any type of fluid cells, and in the particular case of Cartesian fluid cells (such that the cell-face fraction ratios are equal to 1 ), one recovers the finite-difference gradient of the MAC method :

$$
\left[\mathcal{G}^{x} P\right]_{i, j}=\frac{p_{i+1, j}-p_{i, j}}{\frac{1}{2} \Delta x_{i+1}+\frac{1}{2} \Delta x_{i}}\left[\mathcal{M}^{x}\right]_{\mathrm{P}}(i, j),
$$

where $\left[\mathcal{M}^{x}\right]_{\mathrm{P}}(i, j)=\left(\frac{1}{2} \Delta x_{i+1}+\frac{1}{2} \Delta x_{i}\right) \Delta y_{j}$ for the Cartesian control volume $\Omega_{i, j}^{u}$.

In the cut-cells however, it is not possible to interpret Formulas [25] as finitedifference quotients for $p_{i, j}$ located at the centroids of the cut-cells. Instead, the LS-STAG discretization has much in common with the P1 nonconforming/P0 finite element method, where the pressure is approximated with a piecewise constant polynomial with degrees of freedom at the elements centroid (Pironneau, 1989). As a consequence, $p_{i, j}$ is a valid approximation of the pressure anywhere inside cut-cell $\Omega_{i, j}$, even on its solid face. Note that an equivalence of this assumption in a Cartesian mesh is that the pressure gradient is zero at solid boundaries. In the next section, we shall observe that the normal viscous stresses are discretized similarly.

\section{Discretization of the convective fluxes}

For the $x$-momentum Equation [2a], the skew-symmetry property [22] of $\mathcal{C}[\bar{U}]$ imposes upon the discretization of the convective term, that we write in a Cartesian control volume away from the immersed boundary as the following five-point scheme :

$$
\begin{aligned}
\int_{\Gamma_{i, j}^{u}}(\boldsymbol{v} \cdot \boldsymbol{n}) u \mathrm{~d} S \cong & \mathcal{C}[\bar{U}]_{\mathrm{W}}(i, j) u_{i-1, j}+\mathcal{C}[\bar{U}]_{\mathrm{E}}(i, j) u_{i+1, j}+\mathcal{C}[\bar{U}]_{\mathrm{P}}(i, j) u_{i, j} \\
& +\mathcal{C}[\bar{U}]_{\mathrm{S}}(i, j) u_{i, j-1}+\mathcal{C}[\bar{U}]_{\mathrm{N}}(i, j) u_{i, j+1}
\end{aligned}
$$


must verify the following conditions :

$$
\begin{aligned}
& \mathcal{C}[\bar{U}]_{\mathrm{P}}(i, j)=0, \\
& \mathcal{C}[\bar{U}]_{\mathrm{E}}(i, j)=-\mathcal{C}[\bar{U}]_{\mathrm{W}}(i+1, j), \\
& \mathcal{C}[\bar{U}]_{\mathrm{N}}(i, j)=-\mathcal{C}[\bar{U}]_{\mathrm{S}}(i, j+1) .
\end{aligned}
$$

The central discretization of the MAC method trivially verifies these conditions on uniform meshes. Other popular discretizations, such as discretizations of upwind type, are known to violate this condition, resulting in adding artificial viscosity to the scheme.

Skew-symmetric discretizations that verify conditions [27] are now wellestablished methods for high-fidelity simulation of turbulent flows. Morinishi et al. (1998) have build symmetry preserving high-order finite-difference formulas on uniform cartesian grids, and applied them to the LES of turbulent channel flow. Recently, Verstappen et al. (2003) have proposed a skew-symmetric discretization for the DNS of channel flow that enforces conditions [27] on non-uniform Cartesian meshes. This discretization will be the building block of the LS-STAG discretization of the convective term in the cut-cells. For the Cartesian control volume $\Omega_{i, j}^{u}$ of Figure 1, the skew-symmetric discretization consists in writing the convective term as the net flux through its four elementary faces :

$$
\begin{aligned}
\int_{\Gamma_{i, j}^{u}}(\boldsymbol{v} \cdot \boldsymbol{n}) u \mathrm{~d} S= & -\int_{\Gamma_{i, j}^{u, w}}\left(\boldsymbol{v} \cdot \boldsymbol{e}_{x}\right) u \mathrm{~d} y+\int_{\Gamma_{i, j}^{u, e}}\left(\boldsymbol{v} \cdot \boldsymbol{e}_{x}\right) u \mathrm{~d} y \\
& -\int_{\Gamma_{i, j}^{\mathrm{se},} \cup \Gamma_{i+1, j}^{\mathrm{s,w}, w}}\left(\boldsymbol{v} \cdot \boldsymbol{e}_{y}\right) u \mathrm{~d} x+\int_{\Gamma_{i, j}^{\mathrm{n}, \mathrm{e}} \cup \Gamma_{i+1, j}^{\mathrm{n}, \mathrm{w}}}\left(\boldsymbol{v} \cdot \boldsymbol{e}_{y}\right) u \mathrm{~d} x,
\end{aligned}
$$

Each of these terms are discretized with the help of the discrete mass fluxes [11], for example for the east face :

$$
\int_{\Gamma_{i, j}^{u, e}}\left(\boldsymbol{v} \cdot \boldsymbol{e}_{x}\right) u \mathrm{~d} y \cong \frac{\bar{u}_{i, j}+\bar{u}_{i+1, j}}{2} u_{\mathrm{e}},
$$

where $u_{\mathrm{e}}$ is a characteristic value of $u$ on $\Gamma_{i, j}^{u, \mathrm{e}}$, which has to be obtained by interpolation of the discrete velocity unknowns. As observed by Verstappen et al. (2003), the only possible way to verify the skew-symmetric conditions [27] is to use central interpolation with equal weighting :

$$
u_{\mathrm{e}}=\frac{u_{i, j}+u_{i+1, j}}{2} .
$$

Analogous interpolations are obtained on the other faces, for example on the south face :

$$
\int_{\Gamma_{i, j}^{\mathrm{se}} \cup \Gamma_{i+1, j}^{\mathrm{s,w}}}\left(\boldsymbol{v} \cdot \boldsymbol{e}_{y}\right) u \mathrm{~d} x \cong \frac{\bar{v}_{i, j-1}}{2} u_{\mathrm{s}}+\frac{\bar{v}_{i+1, j-1}}{2} u_{\mathrm{s}},
$$


with $u_{\mathrm{s}}=\left(u_{i, j-1}+u_{i, j}\right) / 2$. Now, by using the property of local conservativity of the fluxes through fluid faces :

$$
\begin{aligned}
& \int_{\Gamma_{i, j}^{u, \mathrm{w}}}\left(\boldsymbol{v} \cdot \boldsymbol{e}_{x}\right) u \mathrm{~d} y=\int_{\Gamma_{i-1, j}^{u, \mathrm{e}}}\left(\boldsymbol{v} \cdot \boldsymbol{e}_{x}\right) u \mathrm{~d} y, \\
& \int_{\Gamma_{i, j}^{\mathrm{n}, \mathrm{e}}}\left(\boldsymbol{v} \cdot \boldsymbol{e}_{y}\right) u \mathrm{~d} x=\int_{\Gamma_{i, j+1}^{\mathrm{sec}}}\left(\boldsymbol{v} \cdot \boldsymbol{e}_{y}\right) u \mathrm{~d} x,
\end{aligned}
$$

one gets, after identification with [26], the following coefficients of the discretization :

$$
\begin{aligned}
& \mathcal{C}[\bar{U}]_{\mathrm{P}}(i, j)=\frac{1}{4} \dot{m}^{i, j}+\frac{1}{4} \dot{m}^{i+1, j}, \\
& \mathcal{C}[\bar{U}]_{\mathrm{W}}(i, j)=-\frac{1}{4} \bar{u}_{i-1, j}-\frac{1}{4} \bar{u}_{i, j}, \quad \mathcal{C}[\bar{U}]_{\mathrm{E}}(i, j)=\frac{1}{4} \bar{u}_{i, j}+\frac{1}{4} \bar{u}_{i+1, j}, \\
& \mathcal{C}[\bar{U}]_{\mathrm{S}}(i, j)=-\frac{1}{4} \bar{v}_{i, j-1}-\frac{1}{4} \bar{v}_{i+1, j-1}, \quad \mathcal{C}[\bar{U}]_{\mathrm{N}}(i, j)=\frac{1}{4} \bar{v}_{i, j}+\frac{1}{4} \bar{v}_{i+1, j},
\end{aligned}
$$

which verifies the antisymmetry conditions [27] when the discrete continuity equation is verified in $\Omega_{i, j}$ and $\Omega_{i+1, j}$. Any type of interpolation other than [30], for example an upwind discretization, would violate theses conditions.

In the cut-cells, the skew-symmetric discretization given by [26], [33] must be modified in order to take into account the boundary conditions on the immersed boundary. This discretization would prove to be more complicated to build than for the pressure gradient, because we could not obtain a unique formula which would be valid for any type of cut cells : instead, the discretization should be constructed in each of the half generic control volumes of Figure 3 such as the skew-symmetry condition [22] be verified for any combinations of these half control volumes.

Let us consider the case of the control volume $\Omega_{i, j}^{u}$ of Figure 2, whose north solid boundary $\Gamma_{i, j}^{\mathrm{ib}, \mathrm{e}} \cup \Gamma_{i+1, j}^{\mathrm{ib}, \mathrm{w}}$ is built from two halves of trapezoidal cut-cells. For this control volume, the discretization of the convective term must take the form :

$$
\begin{aligned}
\int_{\Gamma_{i, j}^{u}}(\boldsymbol{v} \cdot \boldsymbol{n}) u \mathrm{~d} S \cong & \mathcal{C}[\bar{U}]_{\mathrm{W}}(i, j) u_{i-1, j}+\mathcal{C}[\bar{U}]_{\mathrm{E}}(i, j) u_{i+1, j}+\mathcal{C}[\bar{U}]_{\mathrm{P}}(i, j) u_{i, j} \\
& +\mathcal{C}[\bar{U}]_{\mathrm{S}}(i, j) u_{i, j-1}+S_{i, j}^{\mathrm{ib}, \mathrm{c}}
\end{aligned}
$$

where $\mathcal{C}[\bar{U}]_{\mathrm{N}}(i, j)$ is discarded since the velocity unknown $u_{i, j+1}$ does not exist in the fluid domain. The skew-symmetry condition [22] reads for this control volume :

$$
\mathcal{C}[\bar{U}]_{\mathrm{P}}(i, j)=0, \quad \mathcal{C}[\bar{U}]_{\mathrm{E}}(i, j)=-\mathcal{C}[\bar{U}]_{\mathrm{W}}(i+1, j) .
$$


The discretization that verifies these conditions is obtained by decomposing the boundary of the control volume as in Equation [7], and writing the convective term as the net flux through each of these faces :

$$
\begin{aligned}
& \int_{\Gamma_{i, j}^{u}}(\boldsymbol{v} \cdot \boldsymbol{n}) u \mathrm{~d} S=-\int_{\Gamma_{i, j}^{u, \mathrm{w}}}\left(\boldsymbol{v} \cdot \boldsymbol{e}_{x}\right) u \mathrm{~d} y+\int_{\Gamma_{i, j}^{u, \mathrm{e}}}\left(\boldsymbol{v} \cdot \boldsymbol{e}_{x}\right) u \mathrm{~d} y \\
& -\int_{\Gamma_{i, j}^{\mathrm{sec}} \cup \Gamma_{i+1, j}^{\mathrm{s,w}}}\left(\boldsymbol{v} \cdot \boldsymbol{e}_{y}\right) u \mathrm{~d} x+\int_{\Gamma_{i, j}^{\mathrm{ib}, \mathrm{e}} \cup \Gamma_{i+1, j}^{\mathrm{ib}, \mathrm{w}}}\left(\boldsymbol{v} \cdot \boldsymbol{n}^{\mathrm{ib}}\right) u \mathrm{~d} S .
\end{aligned}
$$

The fluxes through each of the fluid faces are given by Eqs. [29], [31] and [32], whereas the fluxes through each half of solid face $\Gamma_{i, j}^{\mathrm{ib}, \mathrm{e}}$ and $\Gamma_{i+1, j}^{\mathrm{ib}, \mathrm{w}}$ are discretized separately as :

$$
\begin{gathered}
\int_{\Gamma_{i, j}^{\mathrm{ib}, \mathrm{e}}}\left(\boldsymbol{v} \cdot \boldsymbol{n}_{i, j}^{\mathrm{ib}}\right) u \mathrm{~d} S \cong \frac{\bar{U}_{i, j}^{\mathrm{ib}}}{2}\left(\frac{1}{2} \underline{u_{i, j}}+\frac{1}{2} \underline{\underline{u_{i, j}^{\mathrm{ib}}}}\right), \\
\int_{\Gamma_{i+1, j}^{\mathrm{ib}, \mathrm{w}}}\left(\boldsymbol{v} \cdot \boldsymbol{n}_{i+1, j}^{\mathrm{ib}}\right) u \mathrm{~d} S \cong \frac{\bar{U}_{i+1, j}^{\mathrm{ib}}}{2}\left(\frac{1}{2} \underline{u_{i, j}}+\frac{1}{2} \underline{\underline{u_{i+1, j}^{\mathrm{ib}}}}\right) .
\end{gathered}
$$

In these expressions, the terms underlined once contribute to the diagonal coefficient $\mathcal{C}[\bar{U}]_{\mathrm{P}}(i, j) u_{i, j}$, in order to recover the expression of the discrete continuity in $\Omega_{i, j}$ and $\Omega_{i+1, j}$, whereas the terms which are twice underlined contribute to the source term $S_{i, j}^{\mathrm{ib}, \mathrm{c}}$. As a result, the discretization of the convective term in this control volume is also given by [33], with the exception that :

$$
\mathcal{C}[\bar{U}]_{\mathrm{N}}(i, j)=0, \quad S_{i, j}^{\mathrm{ib}}=\frac{1}{2}\left(\frac{\bar{U}_{i, j}^{\mathrm{ib}}}{2} u_{i, j}^{\mathrm{ib}}+\frac{\bar{U}_{i+1, j}^{\mathrm{ib}}}{2} u_{i+1, j}^{\mathrm{ib}}\right) .
$$

The antisymmetry conditions [35] is thus verified, and we may consider that the source term $S_{i, j}^{\mathrm{ib}} u_{i, j}$ that arises in the kinetic energy budget [24] corresponds to a discrete approximation of the term $\int_{\Gamma^{\mathrm{ib}}}|\boldsymbol{v}|^{2} \boldsymbol{v} \cdot \boldsymbol{n} / 2 \mathrm{~d} S$ written on the solid boundary $\Gamma_{i, j}^{\mathrm{ib}, \mathrm{e}} \cup$ $\Gamma_{i+1, j}^{\mathrm{ib}, \mathrm{w}}$ of the control volume.

For the other types of half control volumes, the expression of the skew symmetric convective flux on the solid boundary is given in Figure 3, while the flux across fluid faces is given by the usual central formulae, e.g. [29] and [31].

\section{Discretization of the viscous terms}

For the $x$-momentum Equation [2a], the viscous terms written in control volume $\Omega_{i, j}^{u}$ reads :

$$
\int_{\Gamma_{i, j}^{u}} \nabla u \cdot \boldsymbol{n} \mathrm{d} S=\int_{\Gamma_{i, j}^{u}} \frac{\partial u}{\partial x} \boldsymbol{e}_{x} \cdot \boldsymbol{n} \mathrm{d} S+\int_{\Gamma_{i, j}^{u}} \frac{\partial u}{\partial y} \boldsymbol{e}_{y} \cdot \boldsymbol{n} \mathrm{d} S
$$


We aim to discretizing these terms in the cut-cells such that the simplicity of the 5point structure of the MAC method be preserved. In this respect, we had to make a distinction between the discretization of the normal stress flux $\int_{\Gamma_{i, j}^{u}} \partial u / \partial x \boldsymbol{e}_{x} \cdot \boldsymbol{n} \mathrm{d} S$ and the shear stress flux $\int_{\Gamma_{i, j}^{u}} \partial u / \partial y \boldsymbol{e}_{y} \cdot \boldsymbol{n} \mathrm{d} S$ in the cut-cells.

For the normal stress flux, a geometry-based formula would consist in writing this term as the net flux through the east $\Gamma_{i, j}^{u, \mathrm{e}}$ and west $\Gamma_{i, j}^{u, \mathrm{w}}$ faces, and then discretize each of these terms with a differential quotient, for example :

$$
\int_{\Gamma_{i, j}^{u, \mathrm{w}}} \frac{\partial u}{\partial x} \boldsymbol{e}_{x} \cdot \boldsymbol{n} \mathrm{d} S \cong \Delta y_{i, j}^{u, \mathrm{w}} \frac{u_{i, j}-u_{i-1, j}}{\Delta x_{i}} .
$$

with the area $\Delta y_{i, j}^{u, \mathrm{w}}$ yet to be defined. All our efforts in this direction gave disappointing results in terms of numerical accuracy. The reason is that the LS-STAG mesh is not admissible in the sense of Eymard et al. (2000) for the normal stresses : the line joining the location of $u_{i-1, j}$ and $u_{i, j}$ is not orthogonal to the face $\Gamma_{i, j}^{u, \mathrm{w}}$ in the trapezoidal cell $\Omega_{i, j}$ of Figure 2. This feature is also observed for the other types of cut-cells (see Figure 3 (a) and (c)), and has the consequence to render approximation [40] non consistent and thus to yield large numerical errors.

In order to improve the consistency of this term, and to retain the simplicity of a 5-point stencil, we use the fact that the discretization of the normal stresses and pressure should be consistent, as stated above, and thus the normal stress flux shall be discretized with an expression similar to the pressure gradient [25a] :

$$
\int_{\Gamma_{i, j}^{u}} \frac{\partial u}{\partial x} \boldsymbol{e}_{x} \cdot \boldsymbol{n} \mathrm{d} S \cong \theta_{i, j}^{u} \Delta y_{j}\left(\left.\frac{\partial u}{\partial x}\right|_{i+1, j}-\left.\frac{\partial u}{\partial x}\right|_{i, j}\right) .
$$

The discretization has to be completed with a differential quotient for $\partial u /\left.\partial x\right|_{i, j}$. This quotient is constructed by requiring that Green's theorem :

$$
\int_{\Omega_{i, j}}\left(\frac{\partial u}{\partial x}+\frac{\partial v}{\partial y}\right) \mathrm{d} V=\int_{\Gamma_{i, j}} \boldsymbol{v} \cdot \boldsymbol{n} \mathrm{d} S
$$

be valid at the discrete level in a cut-cell, since it is trivially verified by the MAC method in a Cartesian cell. After a straightforward discretization of the integrals and comparison with the continuity Equation [12], one gets :

$$
\left.\frac{\partial u}{\partial x}\right|_{i, j} \cong \frac{\theta_{i, j}^{u} u_{i, j}-\theta_{i-1, j}^{u} u_{i-1, j}+\left(\theta_{i-1, j}^{u}-\theta_{i, j}^{u}\right) u_{i, j}^{\mathrm{ib}}}{V_{i j} / \Delta y_{j}} .
$$

This expression is valid for any type of cut-cells, and reduces to the usually finitedifference quotient for a Cartesian fluid cell.

In contrast, the discretization of the shear stress $\int_{\Gamma_{i, j}^{u}} \partial u / \partial y \boldsymbol{e}_{y} \cdot \boldsymbol{n} \mathrm{d} S$ is much simpler because the LS-STAG mesh is admissible in the $y$ direction for this term. 
Thus, we can write it as the net flux through the north and south faces, for example far from the immersed boundary :

$$
\int_{\Gamma_{i, j}^{u}} \frac{\partial u}{\partial y} \boldsymbol{e}_{y} \cdot \boldsymbol{n} \mathrm{d} S=\int_{\Gamma_{i, j}^{\mathrm{n}, \mathrm{e}} \cup \Gamma_{i+1, j}^{\mathrm{n}, \mathrm{w}}} \frac{\partial u}{\partial y} \mathrm{~d} x-\int_{\Gamma_{i, j}^{\mathrm{se}} \cup \Gamma_{i+1, j}^{\mathrm{s}, \mathrm{w}}} \frac{\partial u}{\partial y} \mathrm{~d} x
$$

and the differentiation of the interpolation polynomial of $u\left(x_{i}, \cdot\right)$ in the vertical direction leads to finite difference-like formula, for example :

$$
\begin{aligned}
& \int_{\Gamma_{i, j}^{\mathrm{ne}} \cup \Gamma_{i+1, j}^{\mathrm{n}, \mathrm{w}}} \frac{\partial u}{\partial y} \mathrm{~d} x \cong \\
&\left(\frac{1}{2} \theta_{i, j}^{v} \Delta x_{i}+\frac{1}{2} \theta_{i+1, j}^{v} \Delta x_{i+1}\right) \frac{u_{i, j+1}-u_{i, j}}{\frac{1}{2} \theta_{i, j+1}^{u} \Delta y_{j+1}+\frac{1}{2} \theta_{i, j}^{u} \Delta y_{j}},
\end{aligned}
$$

for the flux on the north face. This formula is valid if $u_{i, j+1}$ is present in the fluid domain, i.e. if $\theta_{i, j+1}^{u}>0$ : this is the case of the Cartesian cell of Figure 1 (b), and the cut-cells in Figure 3 (a) et (b).

In the case where the north face is solid and thus $u_{i, j+1}$ does not exist (case where $\theta_{i, j+1}^{u}>0$, for the cut-cells of Fig . 3 (c)-(f)), the formula takes into account the boundary conditions in the fashion of the Ghost Fluid Method for elliptic equations (Gibou et al., 2002) :

$$
\int_{\Gamma_{i, j}^{\mathrm{ib}, \mathrm{e}} \cup \Gamma_{i+1, j}^{\mathrm{ib}, \mathrm{w}}} \frac{\partial u}{\partial y} \mathrm{~d} x \cong\left(\Delta x_{i, j}^{\mathrm{ib}, \mathrm{e}}+\Delta x_{i+1, j}^{\mathrm{ib}, \mathrm{w}}\right) \frac{u\left(x_{i}, y_{i, j}^{\mathrm{ib}}\right)-u_{i, j}}{\frac{1}{2} \theta_{i, j}^{u} \Delta y_{j}},
$$

where the integration areas $\Delta x_{i, j}^{\mathrm{ib}, \mathrm{e}}$ and $\Delta x_{i+1, j}^{\mathrm{ib}, \mathrm{w}}$ are defined in Figure 3 for each type of cut-cell. As for the discretization of the other fluxes of the Navier-Stokes equations, Formulae [45] and [46] yields the usual discretizations of the original MAC method in a regular fluid cell.

\subsection{Time stepping method and solution of the linear systems}

The time integration of the differential algebraic system [16] and [17] is performed with a semi-implicit projection method based on the AB / BDF 2 scheme. This projection scheme is defined by the following two steps :

$$
\begin{aligned}
\mathcal{M} \frac{3 \widetilde{U}-4 U^{n}+U^{n-1}}{2 \Delta t}+2 \mathcal{C}\left[\bar{U}^{n}\right] U^{n} & -\mathcal{C}\left[\bar{U}^{n-1}\right] U^{n-1} \\
& -\mathcal{D}^{\mathrm{T}} P^{n}-\frac{1}{\operatorname{Re}} \mathcal{K} \widetilde{U}=-S^{\mathrm{ib}, n+1},
\end{aligned}
$$


where $\widetilde{U}$ is a prediction of the velocity at time $t_{n+1}=(n+1) \Delta t$, then :

$$
\begin{aligned}
& \frac{3}{2} \mathcal{M} \frac{U^{n+1}-\widetilde{U}}{\Delta t}-\mathcal{D}^{\mathrm{T}}\left(P^{n+1}-P^{n}\right)=0 \\
& \mathcal{D} U^{n+1}+\bar{U}^{\mathrm{ib}, n+1}=0 .
\end{aligned}
$$

Numerical tests by Botella (2002) shows that this scheme is $\mathcal{O}\left(\Delta t^{2}\right)$ accurate for both velocity and pressure.

The projection step [48] leads to solving following Poisson equation for the pressure potential $\Phi=2 \Delta t\left(P^{n+1}-P^{n}\right) / 3$ :

$$
\mathcal{A} \Phi=\mathcal{D} \widetilde{U}+\bar{U}^{\mathrm{ib}, n+1}, \quad \mathcal{A} \equiv-\mathcal{D} \mathcal{M}^{-1} \mathcal{D}^{\mathrm{T}},
$$

which is a symmetric linear system whose 5-point stencil reads :

$$
\begin{aligned}
& A_{\mathrm{E}}(i, j)=\frac{\left(\theta_{i, j}^{u} \Delta y_{j}\right)^{2}}{\frac{1}{2} V_{i, j}+\frac{1}{2} V_{i+1, j}}, \quad A_{\mathrm{W}}(i, j)=A_{\mathrm{E}}(i-1, j), \\
& A_{\mathrm{N}}(i, j)=\frac{\left(\theta_{i, j}^{v} \Delta x_{i}\right)^{2}}{\frac{1}{2} V_{i, j}+\frac{1}{2} V_{i, j+1}}, \quad A_{\mathrm{S}}(i, j)=A_{\mathrm{N}}(i, j-1), \\
& A_{\mathrm{P}}(i, j)=-A_{\mathrm{E}}(i, j)-A_{\mathrm{W}}(i, j)-A_{\mathrm{N}}(i, j)-A_{\mathrm{S}}(i, j) .
\end{aligned}
$$

In the case of a Cartesian fluid cell, the usual pressure equation of the MAC method is recovered. We mention that the pressure Equation [49] is valid in the whole computational domain, but the solution in the fluid domain is totally decoupled from the one in the solid domain. More precisely, the linear system in the fluid cells has a rank one deficiency, while in the solid cells the system reads :

$$
0 \times \Phi_{i, j}=0,
$$

showing that the pressure is defined up to a different additive constant in each domain. In order to alleviate these indeterminacies in actual computations, we add to the diagonal coefficient $A_{\mathrm{P}}(i, j)$ a small real constant $\epsilon$ whose magnitude has the order of the machine roundoff level, and we solve [49] in the whole computational domain : it amounts in letting the computer roundoff sets the arbitrary pressure levels. With this technique borrowed from Yeckel et al. (1999), the pressure equation is solved simultaneously in both fluid and solid domains with any iterative solver for elliptic equations on Cartesian grids, where no modifications are needed for taking the immersed boundary into account. In the computations we present in the following section, we have used the black-box multigrid/BiCGSTAB solver of van Kan et al. (2000), and we typically observed that the pressure equation was solved in 2-3 iterations. 


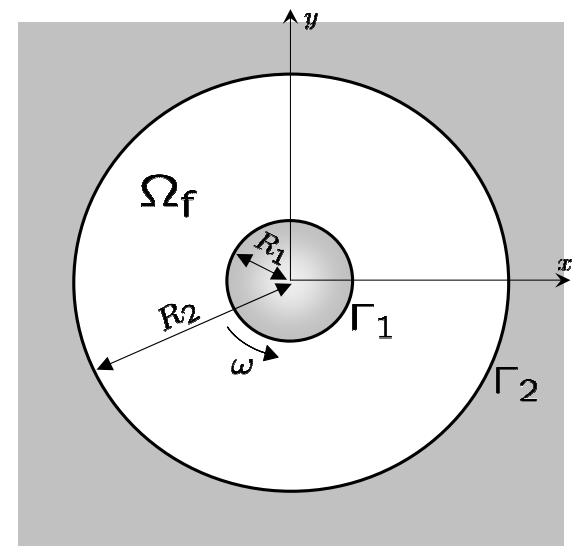

(a)

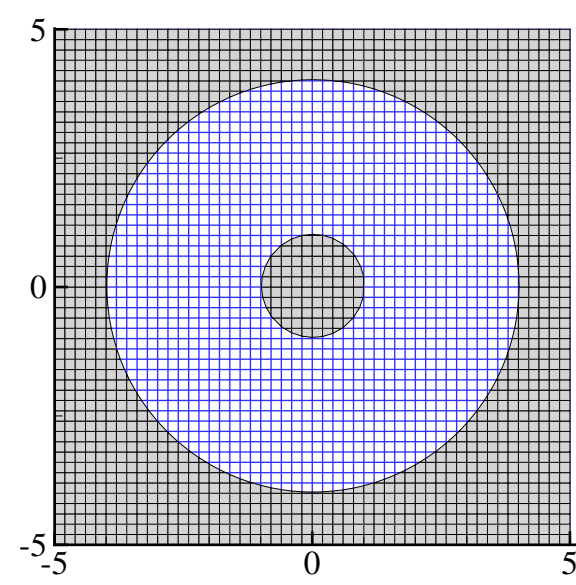

(b)

Figure 4. Geometry and computational domain for the Taylor-Couette flow. (a) : the fluid domain $\Omega_{\mathrm{f}}$ is confined between two concentric cylinders $\Gamma_{1}$ and $\Gamma_{2}$ of center $\left(x_{\mathrm{c}}, y_{\mathrm{c}}\right)$, radius $R_{1}$ and $R_{2}=4 R_{1}$ respectively, where only the inner cylinder $\Gamma_{1}$ moves with the angular velocity $\omega$. $(b)$ : the LS-STAG mesh for $R_{1}=1$ and $N=50$ cells in each direction

\section{Numerical results}

\subsection{Taylor-Couette flow}

First, the spatial accuracy of the LS-STAG method is assessed on the TaylorCouette flow between two concentric circular cylinders, as described in Figure 4 (a). The flow dynamics is governed by the Taylor Number $T a$, which is the ratio between the centrifugal force and the viscous force :

$$
T a=\frac{\omega^{2}\left(\frac{R_{1}+R_{2}}{2}\right)\left(R_{2}-R_{1}\right)^{3}}{\nu^{2}},
$$

where $\nu$ is the kinematic viscosity of the fluid. Below the stability threshold $T a_{\mathrm{c}}=$ 1712 , the flow is steady, stable and purely orthoradial, and the analytical solution is given by e.g. Guyon et al. (2001).

In order to build the level-set function $\phi(x, y)$ that represents the fluid domain $\Omega_{\mathrm{f}}$, we have used the Constructive Solid Geometry (CSG) method for constructing complex domains out of basic geometries such as circles, hyperplanes, spheres, etc., which are sufficiently simple for having an analytical expression for their level-set function (Hart, 1996; Osher et al., 2003). The boolean CSG operations on basic geometries such as intersection, union or complementary part can then be expressed as algebraic operations on their level-set functions (Osher et al., 2003). For example, 
let $\Omega_{1}$ and $\Omega_{2}$ be the inner region of cylinders $\Gamma_{1}$ and $\Gamma_{2}$, whose level-set function is respectively:

$$
\begin{aligned}
& \phi_{1}(x, y)=R_{1}-r \\
& \phi_{2}(x, y)=R_{2}-r
\end{aligned}
$$

Then, the fluid domain of the Taylor-Couette geometry can be constructed as $\Omega_{\mathrm{f}}=$ $\Omega_{2} \backslash \Omega_{1}$, and its level-set function is simply $\phi(x, y)=\max \left(\phi_{2}(x, y),-\phi_{1}(x, y)\right)$.

The computational domain is a square of side length $10 R_{1}$, covered with a uniform mesh of $N$ square computational cells of size $h$ in each direction (see Figure 4 (b)). The center of the concentric cylinders is set at $x_{\mathrm{c}}=0.013, y_{\mathrm{c}}=0.023$ slightly off the center $(x=0, y=0)$ of the computational domain, such as it never corresponds to a corner or centroid of a computational cell. Thus, the numerical error we measure are free of any superconvergence effets, since the natural symmetries of the meshes and the computational solution are broken. For the flow at $\mathrm{Ta}=1000$, we have compared the results of the LS-STAG method with the so-called staircase method, which corresponds to a stepwise approximation of complex geometries with Cartesian cells. This last method is easily obtained from our numerical code by imposing the cell face fraction ratios to be equal to 1 in the cut-cells, while the discretization in the Cartesian cells is unchanged. We will see that even though the cut-cells represent only a small fraction of the computational cells, these modifications will greatly affects the numerical solution in the whole fluid domain.

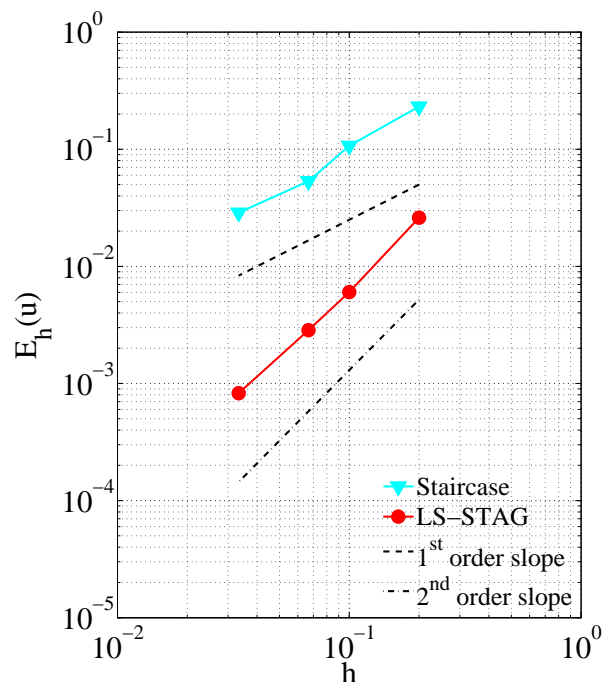

(a)

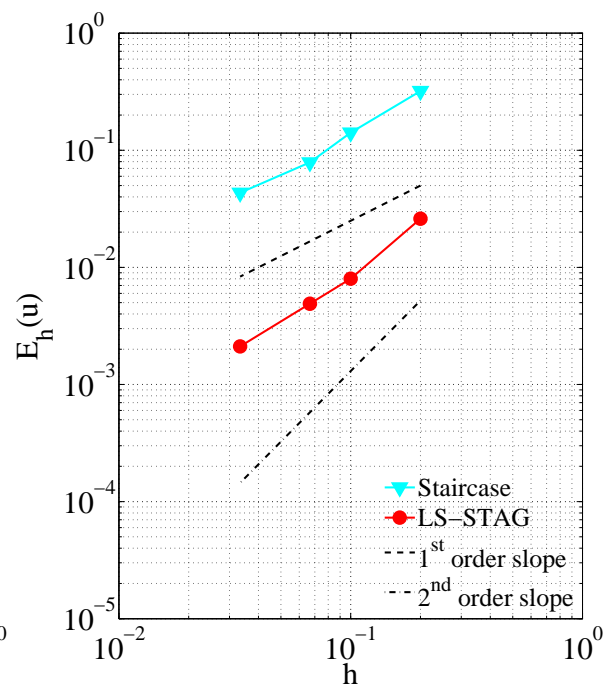

(b)

Figure 5. $L_{\infty}$ norm of the error for the streamwise velocity $u$ versus grid size $h=$ $\frac{10 R_{1}}{N}$. (a) : on $90 \%$ of the fluid domain, $(b)$ : on the whole fluid domain 


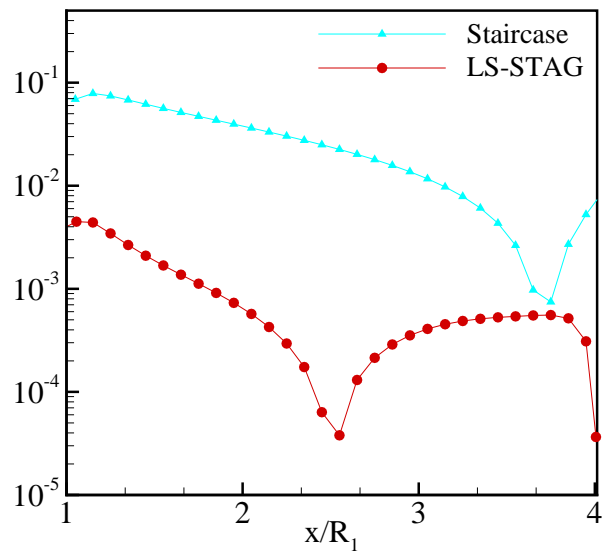

Figure 6. Pointwise error profiles along the horizontal axis $y=0.023$ for the $v$ velocity

For assessing the accuracy of both methods, We have measured the $\mathrm{L}_{\infty}$ spatial error of the $u$ Cartesian component of the velocity up to the cut-cells, ie :

$$
\mathrm{E}_{h}(u)=\max _{\operatorname{CVs} \Omega_{i, j}^{u}}\left|u_{i, j}-u_{\mathrm{ex}}\left(x_{i}, y_{j}+\frac{1}{2} \theta_{i, j}^{u} \Delta y_{j}\right)\right|,
$$

Figure 5 (a) shows the error for the velocity measured on $90 \%$ of the fluid cells away from the immersed boundaries. The LS-STAG method yields a second-order accuracy, much better than the first-order accuracy of the staircase method. When the error of the LS-STAG method is measured on the whole computational domain (Figure 5 (b)), the $\mathrm{L}_{\infty}$ error is slightly higher, showing that maximal error occurs in the vicinity of the cut-cells, and the order of accuracy drops to being superlinear only. This is certainly an effect of the piecewise constant approximation of the normal stresses and the pressure in the cut-cells. Figure 6 shows the pointwise error of the velocity at mesh points along the horizontal radius, for $x \in\left[R_{1}, R_{2}\right]$. Firstly, we observe that the crude treatment of the immersed boundaries for the staircase method pollutes the solution in the whole fluid domain, whereas the pointwise error of the LS-STAG method is much lower, most notably in the inner fluid region. Finally, we mention that a similar trend is observed for the $\mathrm{L}_{\infty}$ error of the pressure : the $\mathrm{L}_{\infty}$ error shows second order accuracy for the LS-STAG method away from the cut-cells, while the staircase method is only first order.

\subsection{Flow past a circular cylinder}

The robustness of the LS-STAG method and its ability to compute unsteady flows at higher Reynolds number is now evaluated on the flow past a circular cylinder in a free stream. The Reynolds number is based on the free stream velocity $U_{\infty}$ and the di- 


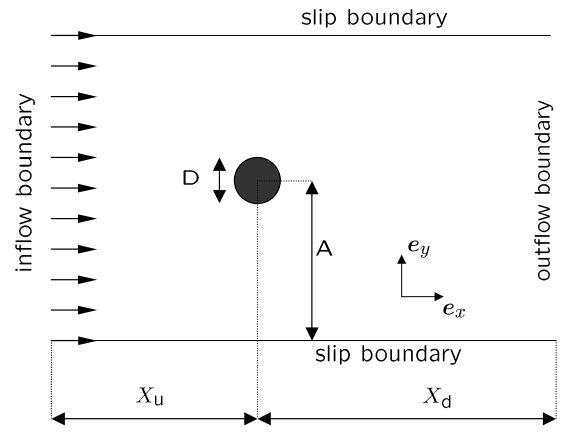

(a)

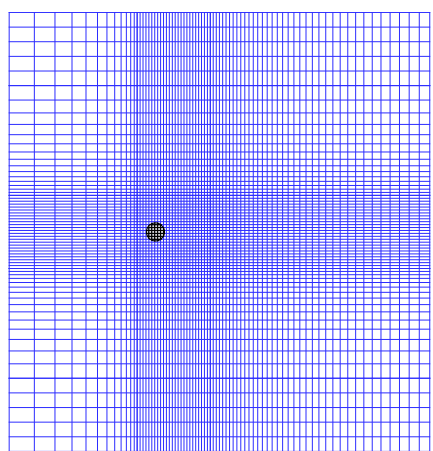

(a)

Figure 7. (a) : Computational domain and $(b)$ : grid for the flow past a circular cylinder

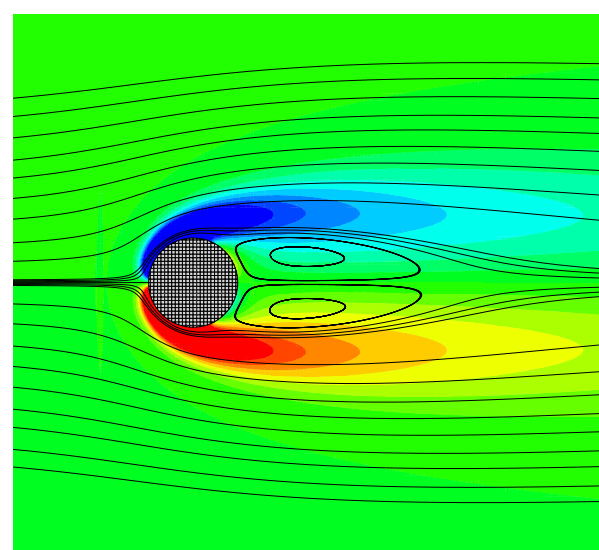

(a)

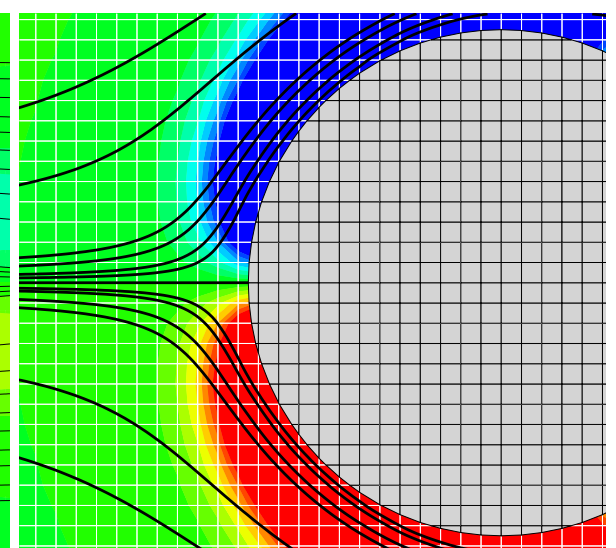

(b)

Figure 8. (a) : vorticity contours and streamlines around a circular cylinder at $R e=$ 40, on M4 mesh. At (b) : close up of the mesh in the vicinity of the cylinder

ameter $D$ of the domain. The flow configuration is described in Figure 7 (a). In all our simulations, the upstream boundary is set at the distance $X_{\mathrm{u}}=8 D$ from the obstacle, the outflow boundary at distance $X_{\mathrm{d}}=15 D$, and the blockage ratio $D / A$ is equal to $1 / 12$. Our previous studies (Cheny et al., 2007) have shown that this computational domain was sufficiently wide for obtaining results independent of the domain size. In order to make a grid refinement study, we used a sequence of non-uniform meshes summarized in Table 1. All these meshes uses a similar block uniform grid of cell size $h / D$ in the wake of the cylinder, as shown in Figure 7 (b). Our simulations with mesh M4 were found to give accurate results for the range of Reynolds number [40 - 1000] 
we considered. The other meshes range from very coarse (mesh M1, used for the steady flow at $\operatorname{Re}=40$ ) to extremely fine (mesh M5 and M6). These last two meshes are mainly used for validating the results obtained on coarser meshes. For comparing our results, we have selected well-established numerical investigations conducted with boundary fitted methods (Henderson, 1997; Bergmann et al., 2007; He et al., 2000), IBM (Linnick et al., 2005) and cut-cell methods (Mittal et al., 2008). Experimental results are taken from the monograph by Zdravkovich (2003).

Table 1. Salient properties of the meshes used for the circular cylinder flow. The percentage of the various type of cells with respect to their total number is given in brackets

\begin{tabular}{ccccccc}
\cline { 3 - 6 } & & \multicolumn{3}{c}{ Type of cells } \\
\hline Mesh & $N_{x} \times N_{y}$ & $h / D$ & $\begin{array}{c}\text { Number } \\
\text { of cells }\end{array}$ & $\begin{array}{c}\text { Cartesian } \\
\text { cells }\end{array}$ & $\begin{array}{c}\text { Solid } \\
\text { cells }\end{array}$ & Cut-cells \\
\hline M1 & $36 \times 34$ & 0.32 & 1224 & $1208(98.7 \%)$ & $4(0.3 \%)$ & $12(1.0 \%)$ \\
M2 & $74 \times 65$ & 0.16 & 4810 & $4767(99.1 \%)$ & $19(0.4 \%)$ & $24(0.5 \%)$ \\
M3 & $150 \times 130$ & 0.08 & 19500 & $19350(99.2 \%)$ & $100(0.5 \%)$ & $50(0.3 \%)$ \\
M4 & $300 \times 260$ & 0.04 & 78000 & $77460(99.3 \%)$ & $440(0.6 \%)$ & $100(0.1 \%)$ \\
M5 & $550 \times 350$ & 0.01 & 192500 & $184452(95.8 \%)$ & $7644(4.0 \%)$ & $404(0.2 \%)$ \\
M6 & $825 \times 524$ & 0.007 & 432300 & $414332(95.8 \%)$ & $17364(4.0 \%)$ & $604(0.2 \%)$ \\
\hline
\end{tabular}

First, we considered the steady flow at $\mathrm{Re}=40$. Figure 8 shows the streamlines and vorticity contours obtained on mesh M4. For producing these figures, the vorticity at cut-cell corners is computed by using the difference formulae for the shear stress (Equation [46] for example). As seen in Figure 8 (b), we may notice the deflection of the streamlines around the cylinder body, showing that the immersed body is correctly taken into account by the LS-STAG method. The accuracy of the computations is assessed by computing the drag coefficient $C_{\mathrm{D}}$ and the length of the recirculation bubble $L_{\mathrm{w}} / D$. The force coefficient is computed by directly approximating the surface integrals of the stress tensor on the immersed boundary, and using formulae [41] and [44] for computing the normal and shear stresses respectively. Table 2 compares the results of the staircase and LS-STAG method against established results from the literature (where the drag coefficient is typically in the range $[1.50-1.54]$ ). The staircase method gives very inaccurate results on the coarser meshes (no recirculation zone is observed on mesh M1), whereas the LS-STAG method gives acceptable results for all meshes, giving asymptotically the values $L_{\mathrm{w}}=2.30 D$ and $C_{\mathrm{D}}=1.51$.

Unsteady flows at $\operatorname{Re}=100,200$, and 1000 have been computed on the M4 and M5 meshes. For breaking the symmetry of the flow and efficiently triggering the vortex shedding, we use as initial condition a discontinuous flow field equal to $U_{\infty}$ in the upper half of the domain, and 0 in the lower half. The flow reaches an asymptotically periodic state at $t U_{\infty} / D=50$, then we starts computing the force coefficients at each time step until $t=350$. The Strouhal number $S t$ is computed as the first harmonic of the power spectrum of the lift coefficient, with a frequency resolution of $\pm 1.67 \times 10^{-3}$ since the length of the time signal is equal to 300 units. Tables 3 
Table 2. Results for the flow at Re $=40$ obtained on the various meshes of Table 1, and comparison with established experimental and computational results from the literature

\begin{tabular}{|c|c|c|c|c|}
\hline & \multicolumn{2}{|c|}{$L_{\mathrm{w}} / D$} & \multicolumn{2}{|c|}{$C_{\mathrm{D}}$} \\
\hline & LS-STAG & staircase & LS-STAC & staircase \\
\hline M1 & 1.715 & 0.000 & 1.599 & 1.185 \\
\hline M2 & 2.095 & 1.319 & 1.547 & 1.402 \\
\hline M3 & 2.219 & 2.041 & 1.551 & 1.545 \\
\hline M4 & 2.300 & 2.101 & 1.500 & 1.527 \\
\hline M5 & 2.299 & 2.226 & 1.508 & 1.559 \\
\hline Experiments (Zdravkovich, 2003) & \multicolumn{2}{|c|}{-} & \multicolumn{2}{|c|}{$1.48-1.70$} \\
\hline Bergmann et al. (2007) & \multicolumn{2}{|c|}{2.26} & \multicolumn{2}{|c|}{1.682} \\
\hline Henderson (1997) & \multicolumn{2}{|c|}{-} & \multicolumn{2}{|c|}{1.545} \\
\hline He et al. (2000) & \multicolumn{2}{|c|}{-} & \multicolumn{2}{|c|}{1.505} \\
\hline Linnick et al. (2005) & \multicolumn{2}{|c|}{2.23} & \multicolumn{2}{|c|}{1.54} \\
\hline Mittal et al. (2008) & \multicolumn{2}{|c|}{-} & \multicolumn{2}{|c|}{1.53} \\
\hline
\end{tabular}

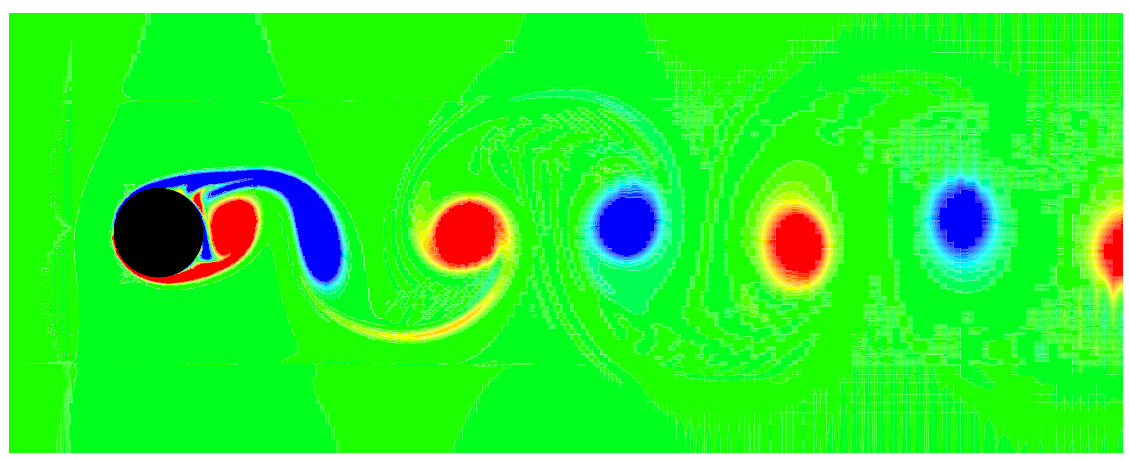

Figure 9. Vorticity contours in the wake of the cylinder at Re $=1000$, computed by the LS-STAG method on the M5 mesh.

and 4 gives salient results computed with the LS-STAG and staircase method. On the M4 mesh for $\mathrm{Re}=100$ and 200 and on the M5 mesh for Re $=1000$, the LS-STAG method gives excellent agreement with the published results. It is also quite remarkable to observe that the staircase method gives marginally acceptable results, even for $\operatorname{Re}=1000$. This is certainly due to the fact that the staircase method inherits the conservation and stability properties of the LS-STAG method, and only the treatment of the immersed boundary differs. 
Table 3. Comparison of time averaged drag coefficient $\bar{C}_{\mathrm{D}}$ and corresponding oscillation amplitude $\pm \Delta C_{\mathrm{D}}$ with established results from the literature

\begin{tabular}{lccc}
\hline $\mathrm{Re}$ & 100 & 200 & 1000 \\
\hline $\mathrm{M} 3$ & $1.352 \pm 0.008$ & $1.350 \pm 0.037$ & $0.979 \pm 0.055$ \\
M4 & $1.322 \pm 0.009$ & $1.332 \pm 0.044$ & $1.493 \pm 0.227$ \\
M5 & $1.317 \pm 0.009$ & $1.327 \pm 0.045$ & $1.530 \pm 0.229$ \\
M6 & $1.314 \pm 0.009$ & $1.324 \pm 0.044$ & $1.524 \pm 0.246$ \\
\hline M4 staircase & $1.323 \pm 0.009$ & $1.346 \pm 0.044$ & $1.610 \pm 0.198$ \\
\hline Experiments (Zdravkovich, 2003) & $1.21-1.41$ & - & - \\
Bergmann et al. (2007) & 1.410 & 1.390 & 1.505 \\
Henderson (1997) & 1.350 & 1.341 & 1.509 \\
He et al. (2000) & 1.353 & 1.356 & 1.519 \\
Linnick et al. (2005) & $1.34 \pm 0.009$ & $1.34 \pm 0.044$ & - \\
Mittal et al. (2008) & 1.35 & - & 1.45 \\
\hline
\end{tabular}

Table 4. Comparison of Strouhal number St with established results from the literature.

\begin{tabular}{lccc}
\hline Re & 100 & 200 & 1000 \\
\hline M3 & 0.170 & 0.200 & 0.260 \\
M4 & 0.170 & 0.200 & 0.247 \\
M5 & 0.170 & 0.200 & 0.241 \\
M6 & 0.170 & 0.200 & 0.241 \\
\hline M4, staircase & 0.177 & 0.207 & 0.251 \\
\hline Experiments (Zdravkovich, 2003) & $0.16-0.17$ & - & - \\
Bergmann et al. (2007) & 0.166 & 0.199 & 0.235 \\
Henderson (1997) & 0.164 & 0.197 & 0.237 \\
He et al. (2000) & 0.167 & 0.198 & 0.239 \\
\hline
\end{tabular}

\section{Concluding remarks}

In this paper, we have developed and analyzed a novel IB/cut-cell method for incompressible viscous flows called the LS-STAG method. The immersed boundary is implicitly represented by its level set, which enables us to calculate efficiently the geometry parameters of the cut-cells. The discretization in the cut-cells has been built by requiring that total kinetic energy is conserved at the discrete level. The LS-STAG discretization preserves the 5-point Cartesian structure of the stencil, resulting in a highly computationally efficient method, and the computations of benchmark flows have shown that the method is a promising kernel for the design and analysis of complex CFD systems. 
This work is also one of the first attempt towards rigourously addressing some important issues for IB computations, such as the conservation of global flow properties (total mass, momentum and kinetic energy) in IB computations, and the consistent implementation of boundary conditions at the immersed boundary. Due to page limitations, we did not include in this paper the proofs of the conservation of total mass and momentum by the LS-STAG method. These issues, as well as novel applications of the LS-STAG method for flows in complex moving geometries, will be discussed in a forthcoming paper.

\section{References}

Bergmann M., Cordier L., Brancher J. P., “ Drag minimization of the cylinder wake by trustregion proper orthogonal decomposition”, in R. King (ed.), Notes on Numerical Fluid Mechanics and Multidisciplinary Design, p. 309-324, 2007.

Botella O., "On a collocation B-spline method for the solution of the Navier-Stokes equations", Computers \& Fluids, vol. 31, p. 397-420, 2002.

Cheny Y., Botella O., “ La méthode LS-STAG : une nouvelle approche de type Frontière Immergée/"Level-Set"' pour le calcul d'écoulements visqueux incompressibles en géométries complexes", 18ème Congrès Français de Mécanique, Grenoble, 27-31 Août, 2007.

Chung M.-H., " Cartesian cut cell approach for simulating incompressible flows with rigid bodies of arbitrary shape", Computers and Fluids, vol. 35, p. 607-623, 2006.

Dröge M., Verstappen R., “ A new symmetry-preserving Cartesian-grid method for computing flow past arbitrarily shaped objects”, Int. J. Numer. Meth. Fluids, vol. 47, p. 979-985, 2005.

Eymard R., Gallouet T., Herbin R., “ Finite volume methods”, in P. Ciarlet, J. L. Lions (eds), Handbook for Numerical Analysis, p. 715-1022, 2000.

Fadlun E. A., Verzicco R., Orlandi P., Mohd-Yusof J., “ Combined immersed-boundary finitedifference methods for three dimensional complex flow simulations", J. Comput. Phys., vol. 161, p. 35-60, 2000.

Gibou F., Fedkiw R. P., Cheng L.-T., Kang M., “ A second-order-accurate symmetric discretization of the Poisson equation on Irregular Domains”, J. Comput. Phys., vol. 176, p. 205-227, 2002.

Guyon E., Hulin J. P., Petit L., Physical Hydrodynamics, Oxford University Press, Oxford, 2001.

Harlow F. H., Welch J. E., “ Numerical calculation of time-dependent viscous incompressible flow of fluid with free surfaces", Phys. Fluids, vol. 8, p. 2181-2189, 1965.

Hart J. C., " Sphere tracing : a geometric method for the antialiased ray tracing of implicit surfaces”, The Visual Computer, vol. 12, p. 527-545, 1996.

He J. W., Glowinski R., Metcalfe R., Nordlander A., Périaux J., “ Active control and drag optimization for flow past a circular cylinder. Part 1. Oscillatory cylinder rotation”, J. Comput. Phys., vol. 163, p. 87-117, 2000.

Henderson R. D., “ Nonlinear dynamics and pattern formation in turbulent wake transition”, $J$. Fluid Mech., vol. 352, p. 65-112, 1997. 
Kang S., Iaccarino G., Moin P., “ Accurate and efficient immersed-boundary interpolations for viscous flows", , In Center for Turbulence Research Briefs, NASA Ames/Stanford University, pp. 31-43, 2004.

Linnick M. N., Fasel H. F., “ A high order immersed interface method for simulating unsteady incompressible flows on irregular domains", J. Comput. Phys., vol. 204, p. 157-192, 2005.

Mittal R., Dong H., Bozkurttas M., Najjar F. M., Vargas A., v. Loebbecke A., “ A Versatile sharp interface immersed boundary method for incompressible flows with complex boundaries", J. Comput. Phys., vol. 227, p. 4825-4852, 2008.

Mittal R., Iaccarino G., “ Immersed Boundary Methods”, Annu. Rev. Fluid Mech., vol. 37, p. 239-261, 2005.

Morinishi Y., Lund T. S., Vasilyev O. V., Moin P., “ Fully conservative higher order finite difference schemes for incompressible flow”, J. Comput. Phys., vol. 143, p. 90-124, 1998.

Muldoon F., Acharya S., " A divergence-free interpolation scheme for the immersed boundary method”, Int. J. Numer. Meth. Fluids., vol. 56, p. 1845-1884, 2008.

Osher S., Fedkiw R. P., Level Set Methods and Dynamic Implicit Surfaces, Springer, New-York, 2003.

Osher S., Sethian J. A., “ Fronts propagating with curvature dependent speed: Algorithms based on Hamilton-Jacobi formulations", Journal of Computational Physics, vol. 79, p. 12-49, 1988.

Patankar S. V., Numerical Heat Transfer and Fluid Flow, McGraw-Hill, New-York, 1980.

Pironneau O., Finite Element Method for Fluids, Wiley, 1989.

Sussman M., Smereka S., Osher S., “ A level set approach for computing solutions to incompressible two-phase flow”, Journal of Computational Physics, vol. 114, p. 146-159, 1994.

van Kan J., Vuik C., Wesseling P., “Fast pressure calculation for 2D and 3D time dependent incompressible flow”, Numer. Linear Algebra Appl., vol. 7, p. 429-447, 2000.

Verstappen R. W. C. P., Veldman A. E. P., “ Symmetry-preserving discretization of turbulent flow”, J. Comput. Phys., vol. 187, p. 343-368, 2003.

Ye T., Mittal R., Udaykumar H. S., Shyy W., “ An Accurate Cartesian Grid Method for Viscous Incompressible Flows with Complex Immersed Boundaries”, J. Comput. Phys., vol. 156, p. 209-240, 1999.

Yeckel A., Derby J. J., “ On setting a pressure datum when computing incompressible flows”, International Journal for Numerical Methods in Fluids, vol. 29, p. 19-34, 1999.

Zdravkovich M. M., Flow Around Circular Cylinders. Volume 1 : Fundamentals, Oxford University Press, Oxford, 2003.

Received: 5 May 2008

Accepted: 6 January 2009 
